\title{
Estimación de la temperatura superficial en imágenes históricas Landsat 5 mediante corrección atmosférica monocanal en el térmico para la cuenca del río Tempisque-Bebedero (Costa Rica)
}

\section{Estimation of the surface temperature in Landsat 5 historical images by means of single-channel atmospheric correction in the thermal for the Tempisque-Bebedero river basin (Costa Rica)}

\author{
Rubén Martínez-Barbáchano ${ }^{I}$ \\ Universidad de Costa Rica, Costa Rica
}

\begin{abstract}
Resumen
En las últimas décadas la utilización de imágenes satelitales se ha incrementado al punto de constituir una herramienta fundamental en el monitoreo de procesos ambientales y de cambio global. En tal sentido, la importancia histórica de la familia de satélites Landsat marca un hito en 1985 con la introducción del sensor Tematic Mapper, que ofrecía la posibilidad a los usuarios de adquirir información de una base histórica de más de 25 años de imágenes térmicas a 120 metros de resolución espacial. El presente trabajo se presenta como una metodología de obtención de temperatura superficial a partir de una serie temporal de imágenes Landsat-5 TM adquiridas en el año 2001 sobre el Pacífico norte de Costa Rica. Se presentan los distintos algoritmos que pueden utilizarse para la estimación de temperatura superficial a partir de datos Landsat con el método monocanal por la función inversa de Planck. Aspectos como la transmisividad media de la atmósfera, la radiancia ascendente y descendente, o la emisividad de la superficie terrestre fueron tomados en cuenta, previamente al cálculo de la temperatura de brillo de las imágenes.
\end{abstract}

Palabras clave: Teledetección, infrarrojo térmico, emisividad, temperatura superficial

1 Máster. Universidad de Costa Rica, Costa Rica. Correo electrónico: ruben.martinezbarbachano@ucr.ac.cr https://orcid.org/0000-0002-9176-3112 


\begin{abstract}
In recent decades, the use of satellite imagery has increased to the point of being a fundamental tool in the monitoring of environmental processes and global change. In this sense, the historical importance of the Landsat family of satellites marks a milestone in 1985 with the introduction of the Tematic Mapper sensor, which offered users the possibility of acquiring information from an over 25 -year historical database of thermal imagery at 120 meters of spatial resolution. This paper is presented as a methodology for obtaining surface temperature data from a time series of Landsat-5 TM images acquired in 2001 on the North Pacific Region of Costa Rica. The different algorithms that can be used for the estimation of surface temperature from Landsat data via the single-channel method by way of the inverse Planck function are presented. Prior to the calculation of the brightness temperature of the images, aspects such as average atmospheric transmissivity, ascending and descending radiance or earth's surface emissivity were taken into account.
\end{abstract}

Keywords: Remote sensing; Thermal infrared; Emissivity; Surface temperature

\title{
Introducción
}

La teledetección espacial ha experimentado un renovado impulso en las últimas décadas, con nuevos sensores y plataformas que han permitido la obtención de magnitudes o variables geofísicas de enorme valor geográfico. Dentro del amplio abanico de dichas variables, la temperatura terrestre es el principal objeto de estudio de la teledetección térmica. De hecho, la temperatura superficial es una fuente fundamental de información, tanto cualitativa como cuantitativa, acerca de los procesos que ocurren en la superficie terrestre, permitiendo por tanto su caracterización, análisis y modelización (Quattrochi y Luvall, 2000).

Las imágenes en infrarrojo térmico de media resolución permiten, además, estimar otras variables como la evapotranspiración a partir de la ecuación de balance energético, de gran utilidad en la gestión del recurso hídrico.

La puesta en órbita en 1982 del sensor Thematic Mapper (TM) a bordo de la plataforma Landsat-4 y posteriormente (en 1984) con la plataforma Landsat-5, ha permitido el acceso a una base histórica de más de 25 años de imágenes térmicas. El lanzamiento en 1999 de la plataforma Landsat-7 con el sensor Enhanced Thematic Mapper plus (ETM+) permitió el acceso a imágenes térmicas con una resolución de 60 metros, aunque un fallo técnico del sensor limitó severamente su uso, por lo que fue sustituido en 2013 por los instrumentos OLI y TIRS de la plataforma Landsat 8. 


\section{Marco conceptual}

Teledetección térmica es un término que hace referencia a las medidas obtenidas por un sensor ubicado a bordo de una plataforma (un avión o satélite) que capta la radiación electromagnética emitida por la superficie terrestre en la región del infrarrojo térmico, típicamente entre los 8 y 14 $\mu \mathrm{m}$ (Sobrino, et al. 2000).

En el intervalo espectral comprendido entre 8 y $14 \mu \mathrm{m}$ (infrarrojo térmico) se produce el $80 \%$ de la emisividad de la superficie terrestre. En dicho intervalo es donde, además, la transmisividad atmosférica es más elevada y por tanto, donde se ubican las medidas que diferentes sensores toman a bordo de las misiones de los satélites (Pérez y Muñoz, 2002).

La atenuación de la señal en dicha región del espectro se debe a la absorción de aerosoles, pero se trata de una cantidad despreciable debido a que las concentraciones de vapor de agua $\left(\mathrm{H}_{2} \mathrm{O}\right)$ ozono $\left(\mathrm{O}_{3}\right)$ y dióxido de carbono $\left(\mathrm{CO}_{2}\right)$ se encuentran en menor proporción (Prata et al., 1993).

Sin embargo, la transmisión atmosférica de la radiación se ve afectada por el vapor de agua cinco veces más que por el resto de los gases juntos, absorbiendo la radiación de onda larga del infrarrojo térmico y onda corta en la región de las microondas (desde los $22 \mu \mathrm{m}$ a $1 \mathrm{~mm}$ ). En cuanto al dióxido de carbono, este absorbe fuertemente la radiación en la región del infrarrojo lejano del espectro electromagnético, mientras que el ozono lo hace para longitudes de ondas más cortas y energéticas (Atkins \& Jones, 2006).

En consecuencia, la estimación de la temperatura superficial está condicionada, principalmente, por el contenido de vapor de agua de la atmósfera (especialmente sensible en la zona intertropical) y la emisividad de la superficie estudiada. Por todo ello, una correcta estimación de la temperatura superficial implica necesariamente la consideración y corrección de estos factores.

La radiación emitida por una superficie a una determinada longitud de onda, dentro del rango infrarrojo térmico, es función no sólo de su temperatura, sino que depende también de su emisividad. Es por ello, un parámetro crítico para el estudio del balance radiativo terrestre. La ecuación de la emisividad está representada como la energía emitida por una superficie eficiente (Valor y Caselles, 1996) y viene dada por: 


$$
\varepsilon_{i}=\frac{R_{i(T s)}-L_{i}^{\alpha}}{B_{i(T s)}-L_{i}^{\alpha}}
$$

Ecuación 1. Ecuación de la emisividad (Valor y Caselles, 1996)

Siendo $\varepsilon_{i}$ la emisividad de una superficie (considerando la transmisividad e irradiancia de la atmósfera), $\mathbf{R}_{i(T s)}$ la radiancia espectral emitida por un cuerpo a una determinada longitud de onda y temperatura, $\mathbf{B}_{i(T s)}$ la radiancia emitida por un cuerpo negro a una temperatura dada, medida en campo, y por último $\mathbf{L}_{\mathrm{i} \infty}$ la irradiancia atmosférica.

Se suele definir un cuerpo negro como un emisor perfecto, es decir, con una emisividad de 1; sin embargo, la vegetación tiene valores de emisividad menores, entre 0,97 - 0,985, debido a que parte de la energía incidente es reflejada, absorbida y transmitida en proporciones diferentes, según la longitud de onda (Rubio et al., 2003). Su valor es altamente dependiente de la composición de la superficie, sobre todo con su contenido de humedad, rugosidad y tamaño de sus partículas (Barreto, et al. 2009).

También varía con la longitud de onda en la cual es medida, la resolución y ángulo de observación, dando una idea de la complejidad que representa su estimación. Algunas de las técnicas que se han utilizado para estimar la emisividad de la superficie son:

- $\quad$ Método de emisividad normalizada (NEM) propuesto por Gillespie et al., (1985).

- $\quad$ El índice Independiente de la Temperatura (TISI) propuesta por Becker y Li, (1990).

- La separación de emisividad (TES) propuesta por Gillespie et al. (1999).

- $\quad$ El método basado en índice de vegetación (NDVI) propuesto por Valor y Caselles, (1996) que fue el utilizado en el presente estudio.

El canal térmico (6 para Landsat TM-ETM+) convertido a radiancia se puede transformar a temperatura de brillo efectiva por medio de la 
Estimation of the surface temperature in Landsat 5 historical images by means of single-channel atmospheric correction in the thermal for the Tempisque-Bebedero river basin (Costa Rica)

ecuación de cálculo de la temperatura superficial a partir de los valores de brillo de la imagen térmica, que requiere de dos constantes de calibración como muestra el cuadro 1 :

Cuadro 1. Constantes de calibración de la banda térmica del satélite Landsat

\begin{tabular}{cccc}
\hline Satélites & Bandas & $\mathrm{K}_{1}$ & $\mathrm{~K}_{2}$ \\
\hline & & $\left(\mathrm{W} / \mathrm{m}^{-2} / \mathrm{sr}^{-1} / \mu \mathrm{m}^{-1}\right)$ & Kelvin \\
\hline Landsat 4 & 6 & 671,62 & 1284,30 \\
\hline Landsat 5 & 6 & 607,76 & 1260,56 \\
\hline Landsat 7 & 6 & 666,09 & 1282,71 \\
\hline Landsat 8 & 10 & 774,89 & 1321,08 \\
\hline & 11 & 480,89 & 1201,14 \\
\hline
\end{tabular}

Fuente: Chander et al., 2009.

Por otra parte, el uso de la banda térmica en el estudio de la temperatura superficial de la Tierra requiere de la eliminación de los efectos atmosféricos en dicha longitud de onda. Además, la emisividad de un objeto en el terreno es igualmente atenuada y aumentada por la atmósfera. Por todo ello, resulta imprescindible un conocimiento apropiado de la atmósfera para la estimación de la transmitancia, la radiancia ascendente y la radiancia descendente. Cuando se conocen los valores de estas 3 variables, se puede convertir la radiancia medida en el espacio a la radiancia emitida por una superficie (Barsi et al., 2005).

\section{Área geográfica de estudio}

La cuenca del río Tempisque-Bebedero, en el noroeste de Costa Rica, cubre en total $5455 \mathrm{~km}^{2}$ en la provincia de Guanacaste. La cuenca se encuentra situada $10^{\circ} 58^{\prime} 25^{\prime \prime}$ de latitud norte, $10^{\circ} 06^{\prime} 41^{\prime \prime}$ de latitud sur, $85^{\circ} 46^{\prime} 59^{\prime \prime}$ oeste y $84^{\circ} 49^{\prime} 40^{\prime \prime}$ este. El clima predominante en la cuenca es de tipo tropical con una prolongada estación seca, que va de finales de noviembre a principios de mayo. La estación de lluvias comienza en mayo y se prolonga hasta noviembre. Existe por tanto una fuerte estacionalidad en las precipitaciones, con un valor promedio anual de $1900 \mathrm{~mm}$. En cuanto a las temperaturas, no existe la marcada estacionalidad que se da en las precipitaciones, con un promedio anual de $28{ }^{\circ} \mathrm{C}$. Sin embargo, es habitual 
en plena estación seca que la temperatura promedio ascienda hasta los 34 ${ }^{\circ} \mathrm{C}$ de promedio, con máximos puntuales de hasta $40{ }^{\circ} \mathrm{C}$. Desde un punto de vista geomorfológico se trata de una zona de origen predominantemente sedimentario, y por ello reciente. El complejo Nicoya configura la red de drenaje en virtud de colinas calizas que constituyen un espacio formado fundamentalmente a partir del Mioceno (Denyer, Montero \& Flores, 2014). La existencia de espacios abiertos, llanuras inundables, cultivos y pastizales facilita la obtención de temperatura superficial del suelo, tanto por la homogeneidad de los pixeles obtenidos por el satélite como por la existencia de suelo sin cobertura forestal densa.

\section{Materiales y métodos}

Para el presente trabajo se utilizaron exclusivamente recursos libres online y software de código abierto. Todo el procesamiento de imágenes que sustenta la presente metodología se realizó con la herramienta "calculadora de mapas" de GvSIG 2.4 (figura 1).

Figura 1. Calculadora de mapas de GvSIG

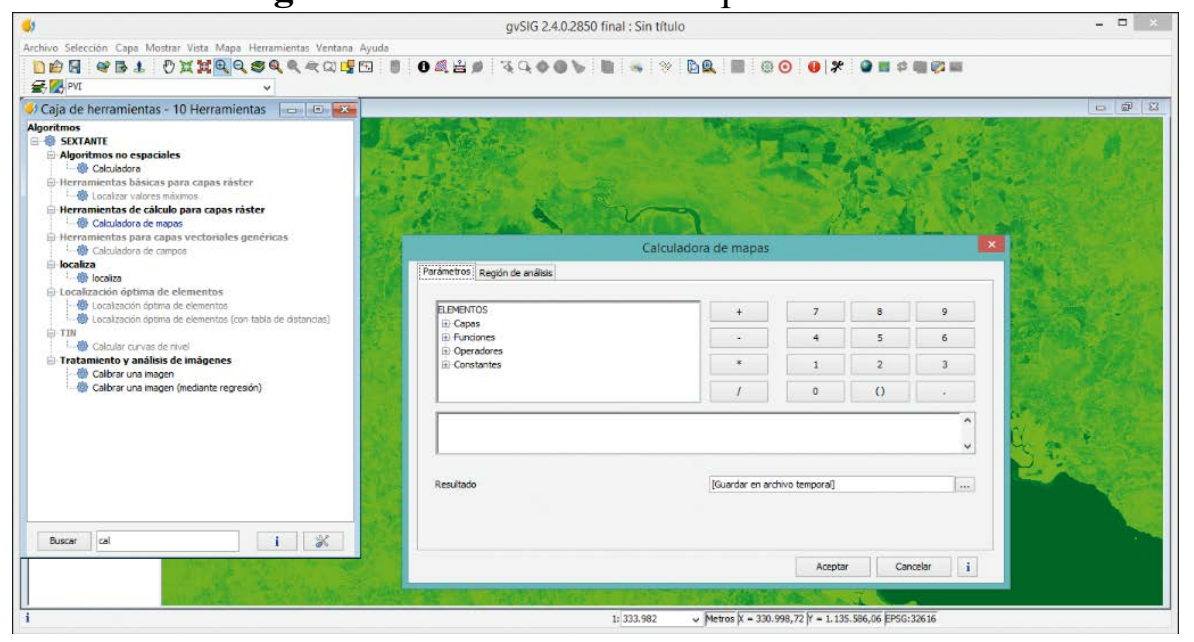

El problema fundamental con la estimación de la temperatura superficial de la Tierra a partir de datos de satélite es la necesidad de corregir en los mismos, los efectos debidos a la absorción atmosférica y a la emisividad de la superficie medida. Para la producción del mapa de emisividad, 
Estimation of the surface temperature in Landsat 5 historical images by means of single-channel atmospheric correction in the thermal for the Tempisque-Bebedero river basin (Costa Rica)

por tanto, se parte del modelo presentado por (Valor y Caselles, 1996). Pero previamente, es conveniente la realización de una corrección DOS1 a las bandas roja e infrarroja de Landsat5, para remover el efecto de la dispersión atmosférica.

Seguidamente, calculamos el índice de vegetación diferencial normalizado, o NDVI (como se muestra en la figura 2) a partir de las bandas 3 y 4, ya corregidas atmosféricamente. La fórmula del NDVI es:

$$
\text { NDVI }=\frac{(\text { NIR }- \text { Red })}{(\text { NIR }+ \text { Red })}
$$

Ecuación 2. Índice de vegetación diferencial normalizado

Figura 2. Índice de Vegetación Diferencial Normalizado o NDVI de la cuenca del Río Tempisque-Bebedero, Costa Rica

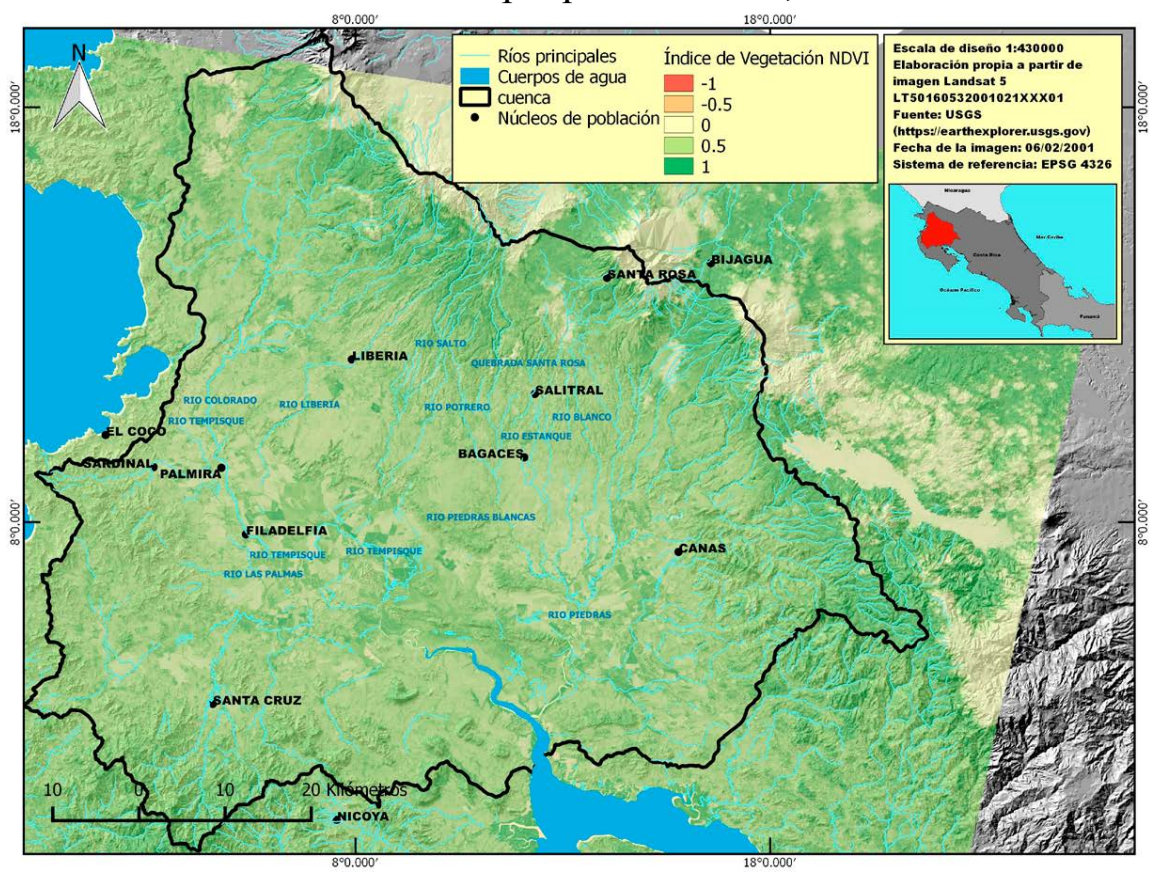

Una vez obtenido el NDVI, se puede utilizar este directamente para calcular la emisividad de los pixeles, o bien se puede generar un PV (Proportion of Vegetation). La ventaja de esta segunda opción es que permite 
aislar la emisividad de las diferentes cubiertas, tomando como referencia la de la vegetación que puede ser calculada a partir de este índice (Carlson \& Ripley, 1997) mostrado en la ecuación 3.

$$
P_{\mathrm{v}}=\left[\frac{\mathrm{NDVI}-\mathrm{NDVI}_{\min }}{\mathrm{NDVI}_{\max }-\mathrm{NDVI}_{\min }}\right]^{2}
$$

Ecuación 3. Proporción de vegetación

Fuente: Carlson \& Ripley, 1997.

Figura 3. Índice de Proporción de Vegetación o PV, de la cuenca del Río Tempisque-Bebedero, Costa Rica

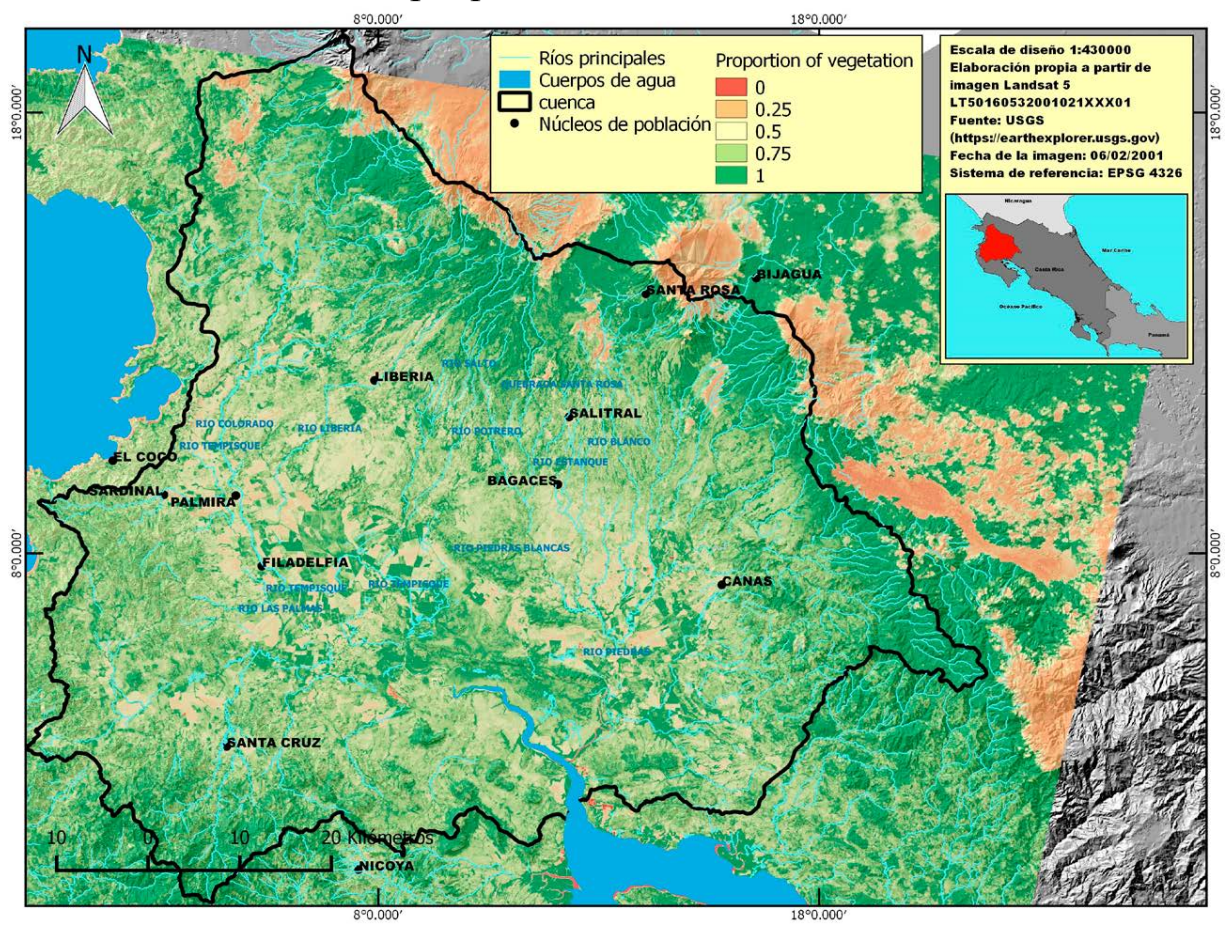

En la figura 3 se puede constatar que la cantidad de superficie cubierta por vegetación es sensiblemente menor en el PVI que en el NDVI.

Seguidamente, se procede a mapear la emisividad de los pixeles a partir de la vegetación, como se muestra en la figura 4. Asumiendo que el 
Estimation of the surface temperature in Landsat 5 historical images by means of single-channel atmospheric correction in the thermal for the Tempisque-Bebedero river basin (Costa Rica)

valor típico de la emisividad en el térmico es de 0.99 para la vegetación, escoger un valor típico de emisividad para el suelo puede ser algo más complejo. Una posibilidad es tomar como referencia el valor propuesto a partir de las 49 muestras de la biblioteca espectral de Aster (http://asterweb.jpl.nasa.gov). En tal caso el valor medio es de 0.973, con una desviación estándar de 0.004 y la expresión final para calcular la emisividad en el monocanal térmico sería la correspondiente con la ecuación 4:

$$
\varepsilon_{\mathrm{TM} 6}=0.004 P_{\mathrm{v}}+0.986
$$

Ecuación 4. Cálculo de emisividad a partir de la proporción de vegetación o $\mathrm{Pv}$

Fuente: Sobrino, Jiménez-Muñoz \& Paolini, 2004.

Figura 4. Mapa de emisividad de la cuenca del Río TempisqueBebedero, Costa Rica

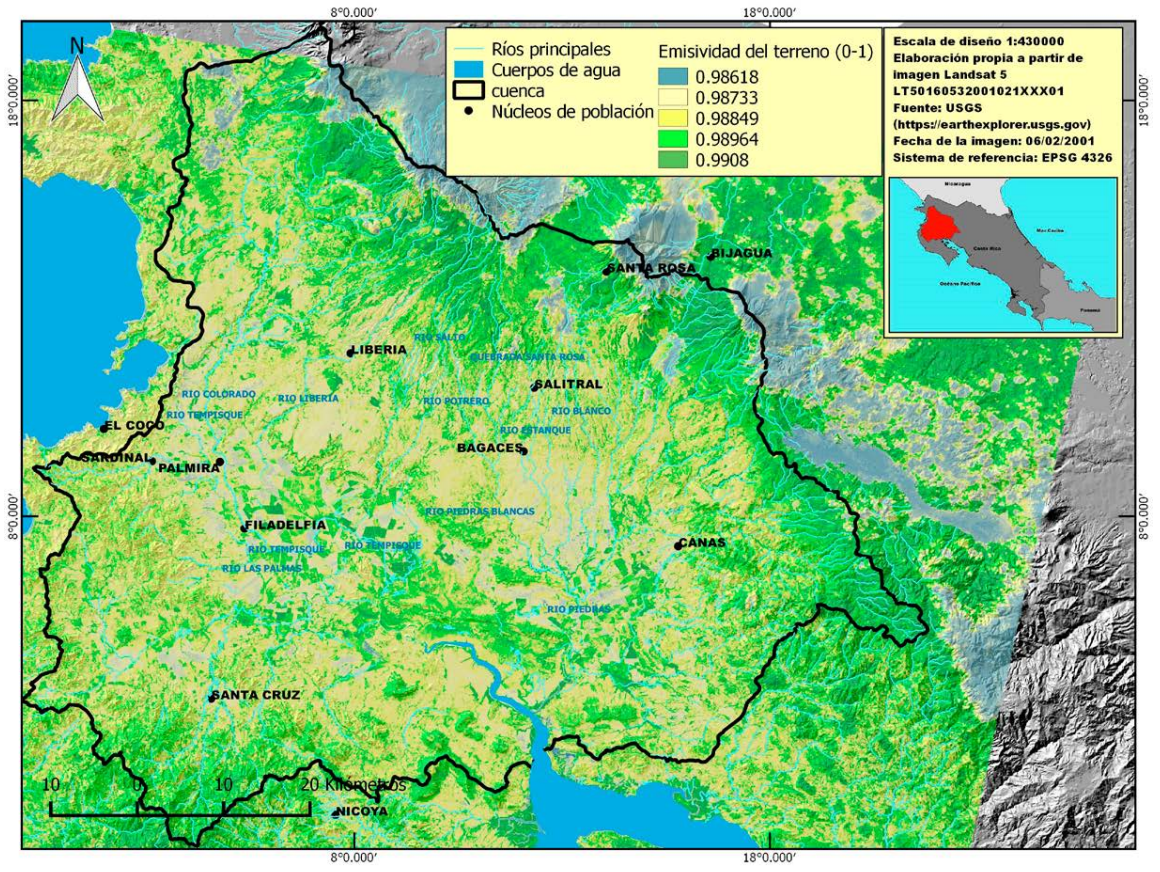

Se va a tomar como referencia el valor de emisividad del pixel correspondiente a la estación meteorológica de Liberia (figura 5) con coordenadas UTM 16 N 1172043 X / 660453 Y 
Figura 5. El valor de emisividad para el pixel de la estación de Liberia es de 0.987321

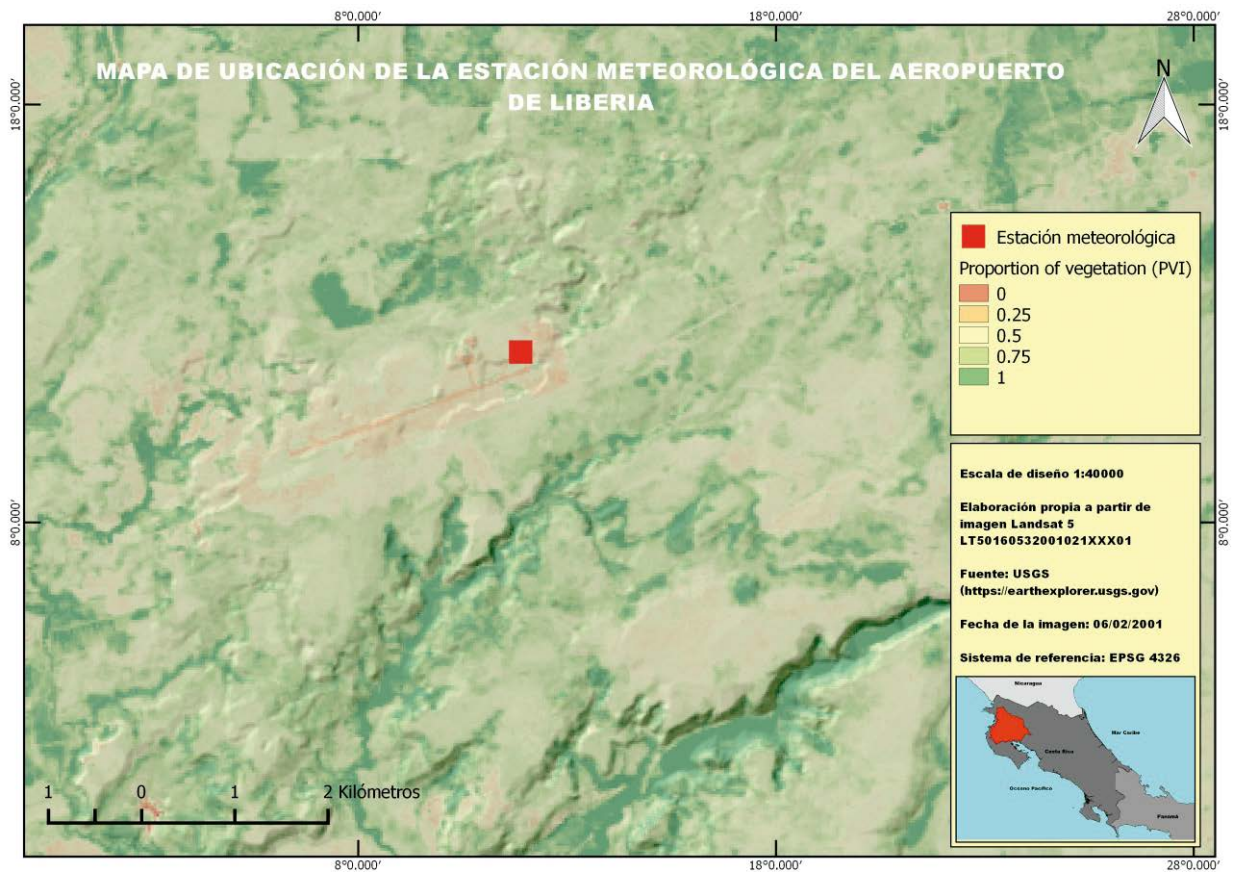

Se calculó la temperatura superficial a partir del método monocanal, basado en la ecuación de transferencia radiativa de Coll, Galve, Sánchez y Caselles (2010). Para ello, se tuvieron en cuenta los aportes de Valor y Caselles (1996) y Chander et al. (2009).

En primer lugar, se realizó una calibración radiométrica para llevar los valores digitales a radiancia espectral $\left(\mathrm{W} / \mathrm{m}^{-2} / \mathrm{sr}^{-1} / \mu \mathrm{m}^{-1}\right)$ como muestra la figura 6. La calibración radiométrica o transformación a valores de radiancia es un paso esencial para poder comparar datos o información de múltiples sensores y plataformas en una escala radiométrica común.

La radiancia se calcula de acuerdo a los metadatos del archivo MTL de la banda 6 , a través de la ecuación 5 :

$$
L_{\lambda}=\left(\frac{L M A X-L M I N}{Q C A L M A X-Q C A L M I N}\right) *(D N-Q C A L M I N)+L M I N
$$

Ecuación 5. Cálculo de radiancia 
Estimation of the surface temperature in Landsat 5 historical images by means of single-channel atmospheric correction in the thermal for the Tempisque-Bebedero river basin (Costa Rica)

Donde $L_{\lambda}$ es la radiancia espectral de la banda térmica que va a ser calculada; $L M A X$ y $L M I N$ son los valores de radiancia máxima y mínima para la banda 6; $Q C A L M A X$ y $Q C A L M I N$ son los valores máximo y mínimo de la radiancia calibrada, es decir, modificados a escala en unidades de números digitales (en función de la resolución radiométrica del sensor) y DN son los niveles digitales (la banda 6 en este caso).

Figura 6. Radiancia espectral de la cuenca del Río Tempisque-Bebedero, Costa Rica

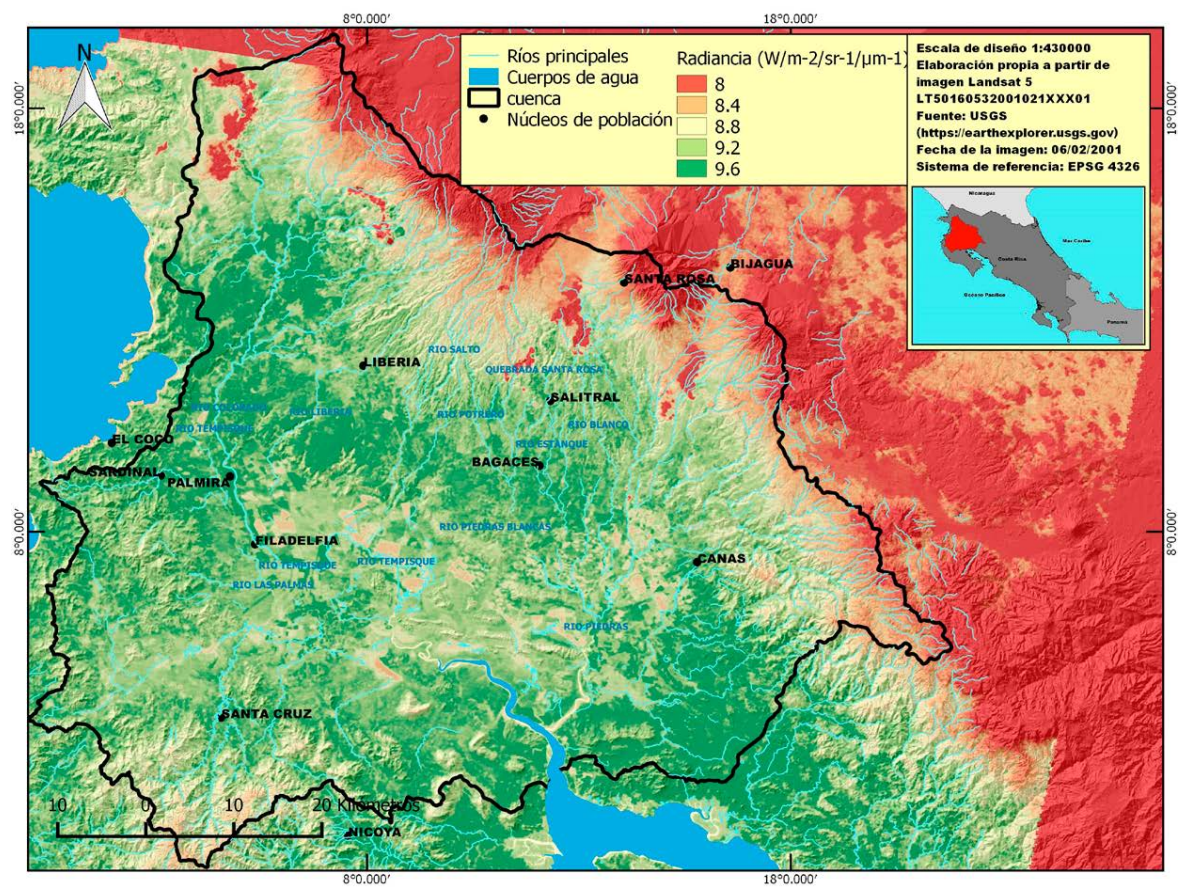

Para calcular la temperatura superficial se consideró la siguiente ecuación de transferencia radiativa de C. Coll, J. M. Galve, J. M. Sánchez y V. Caselles (2010):

$$
B(T)=\frac{L_{\text {sen }}-L^{\uparrow}}{\varepsilon \tau}-\frac{1-\varepsilon}{\varepsilon} L^{\downarrow}
$$

Ecuación 6. Ecuación de transferencia radiativa Fuente: Coll, Galve, Sánchez y Caselles, 2010. 
Donde; $\boldsymbol{L}_{\text {sen }}$ es la radiancia medida por el sensor $\left(\mathrm{W} / \mathrm{m}^{-2} / \mathrm{sr}^{-1} / \mu \mathrm{m}^{-1}\right)$, $\varepsilon$ es la emisividad de la superficie, $\boldsymbol{B}$ es el parámetro derivado de la Ley de Planck calculado a partir de la ecuación de cálculo de temperatura de la superficie terrestre $\left({ }^{\circ} \mathrm{K}\right), \boldsymbol{L} \downarrow$ : radiancia atmosférica descendente $\left(\mathrm{W} / \mathrm{m}^{-2} /\right.$ $\left.\mathrm{sr}^{-1} / \mu \mathrm{m}^{-1}\right), \boldsymbol{\tau}$ : transmisividad atmosférica y $\boldsymbol{L}^{\uparrow}$ : radiancia atmosférica ascendente $\left(\mathrm{W} / \mathrm{m}^{-2} / \mathrm{sr}^{-1} / \mu \mathrm{m}^{-1}\right)$.

La transmisividad de la atmósfera, y la radiancia ascendente y descendente no son parámetros presentes en los metadatos de una imagen satelital. Para su obtención se recurrió a una herramienta operativa en línea de corrección atmosférica que permite calcular la temperatura superficial con un sesgo inferior a $0.5 \pm 0.8^{\circ} \mathrm{K}$ (Barsi y otros, 2005). La herramienta se denomina Atmospheric Correction Parameter Calculator y está disponible en el sitio web; https://atmcorr.gsfc.nasa.gov/.

Figura 7. Para la fecha de la escena Landsat (6 de febrero de 2001) se obtuvieron unos valores de transmisividad atmosférica de 0,54 , una radiancia ascendente de $3,66\left(\mathrm{~W} / \mathrm{m}^{-2} / \mathrm{sr}^{-1} / \mu \mathrm{m}^{-1}\right)$ y una radiancia descendente de 5,50 (W/m-2/sr $\left.1 / \mu \mathrm{m}^{-1}\right)$. Fuente: https://atmcorr.gsfc.nasa.gov/
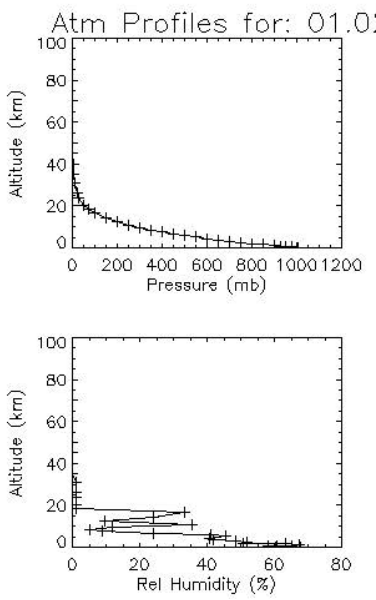

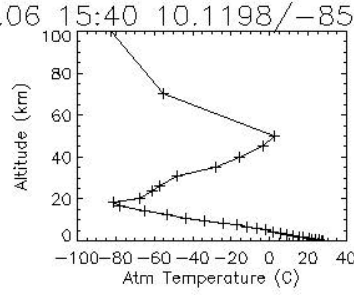

$t=0.54$
Lu $=3.66$ $\mathrm{Ld}=5.50$
Atmospheric Correction Parameter Calculator

Input summary

Date (yyyy-mm-dd): 2001-02-06

Lat/Long: $10.120 /-85.651$

GMT Time: $15: 40$

L5 Spectral Response Curve from handbook

Mid-latitude summer standard atmosphere

Using surface conditions from the model profile

Output summary

Band average atmospheric transmission: 0.54 Effective bandpass upwelling radiance: $3.66 \mathrm{~W} / \mathrm{m}^{\wedge} 2 / \mathrm{sr} / \mathrm{um}$ Effective bandpass downwelling radiance: $5.50 \mathrm{~W} / \mathrm{m}^{2} / \mathrm{sr} / \mathrm{um}$

Barsi y otros (2003) afirman que los valores de temperatura superficial pueden ser estimados con un error de $\pm 2^{\circ} \mathrm{K}$ cuando la atmósfera está relativamente despejada y se conoce la emisividad de la superficie. Esta herramienta utiliza perfiles atmosféricos del National Center for 
Environmental Prediction (NCEP) interpolados a una posición, fecha y hora dadas y el modelo MODTRAN 4 (Berk et al. 1999).

Seguidamente, se aplicó la ecuación 7 de transferencia radiativa propuesta por Coll, Galve, Sánchez y Caselles (2010). El error que introduce esta ecuación se incrementa con el ancho de longitud de onda, la humedad atmosférica y las variaciones de emisividad de la superficie (Coll et al, 2010).

El siguiente paso consistió en aplicar la ecuación 7 de cálculo de temperatura de brillo:

$$
T_{b}=\frac{k_{2}}{\ln \left(\frac{k_{1}}{L_{\mathrm{sen}}}+1\right)}
$$

Ecuación 7. Cálculo de la temperatura a partir de los valores de brillo de la imagen (Chander, 2009)

Donde $k_{2}$ y $k_{1}$ son los coeficientes de calibración, con valores de 1260,56 y $607,76\left(\mathrm{~W} / \mathrm{m}^{-2} / \mathrm{sr}^{-1} / \mu^{-1}\right)$, respectivamente, (Chander et al. 2009) mientras que $L_{\text {sen }}$ es el ráster de radiancia corregida obtenido en el paso anterior. El resultado se obtiene en grados kelvin, que fueron transformados en Celsius.

Figura 8. Temperatura superficial en grados Celsius de la cuenca del río Tempisque-Bebedero Costa Rica

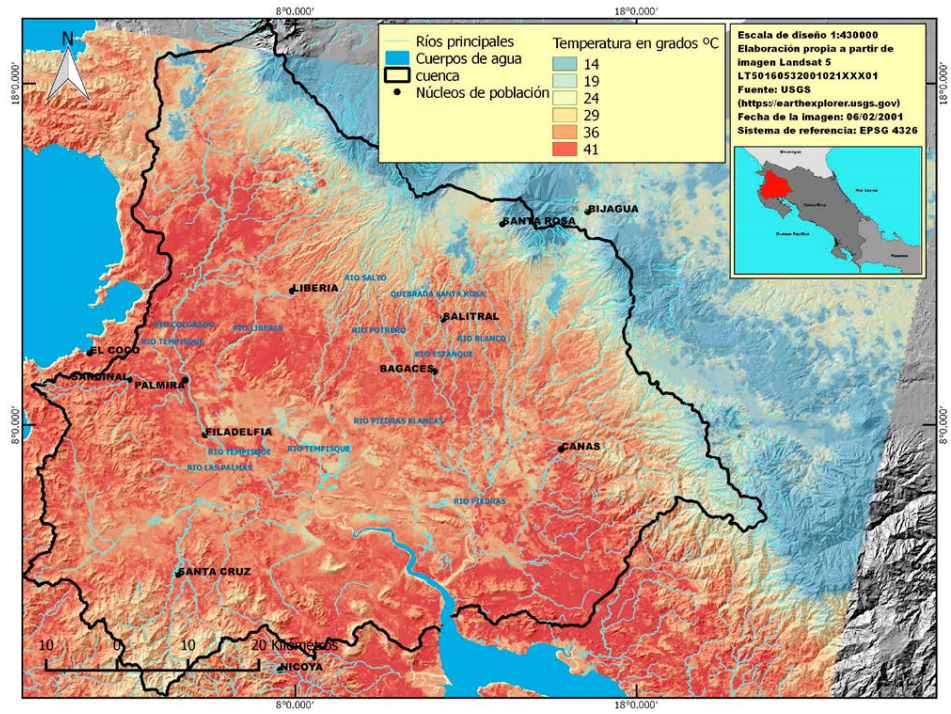




\section{Conclusiones}

La temperatura finalmente obtenida para el aeropuerto de Liberia fue de $44,379^{\circ} \mathrm{C}$ a las 15:40 del 6 de febrero de 2001. De acuerdo con la información proporcionada por el Instituto Meteorológico Nacional de Costa Rica, la temperatura máxima en el aeropuerto de Liberia ese día fue de $34,1^{\circ} \mathrm{C}$. La obtención de la temperatura sin tomar en cuenta parámetros como la emisividad, la transmisividad y la radiancia bidireccional (es decir, aplicando la ecuación 8 directamente sobre la radiancia) genera valores de temperatura más bajos, y por tanto más parecidos a la temperatura del aire a la sombra y a cierta distancia del suelo. Concretamente, en el caso del pixel correspondiente a la estación meteorológica del aeropuerto de Liberia, la temperatura obtenida con este método fue de $32^{\circ} \mathrm{C}$. Considerando la presencia de suelo asfaltado y el hecho de que la temperatura superficial puede superar ampliamente a la temperatura medida a la sombra y a un metro del suelo, la diferencia de $12{ }^{\circ} \mathrm{C}$ parece más que plausible. Por otra parte, cuanto menor es la irradiancia solar y mayor es la velocidad del viento, menores diferencias existen entre estas temperaturas, y por ello más próxima es la temperatura del aire a la temperatura superficial medida desde satélite. Además, estas diferencias también disminuyen a mayor proporción de cobertura vegetal y cuando no existe estrés hídrico (Niclós, et al, 2010).

La existencia de correlaciones entre temperatura del aire y temperatura superficial en diferentes tipos de coberturas de la zona intertropical y en zonas con diferentes relieves debe ser estudiada con mayor profundidad. También se requiere analizar y validar la introducción de nuevos parámetros en las ecuaciones propuestas, con objeto de obtener un algoritmo general que permita estimar la temperatura del aire a partir de la observación desde satélite.

La principal desventaja que presenta esta metodología deriva del hecho de que el valor de emisividad que se incorpora a la ecuación 5 proviene de un pixel del terreno (no de un valor promedio de la imagen) por lo que la estimación del valor de temperatura superficial de la imagen sólo tendría una precisión de $\pm 2^{\circ} \mathrm{K}$ en el pixel del cual se tomó el respectivo valor de emisividad.

Finalmente, la utilidad de esta metodología monocanal de obtención de temperatura superficial permite aproximaciones históricas (como la 
Estimation of the surface temperature in Landsat 5 historical images by means of single-channel atmospheric correction in the thermal for the Tempisque-Bebedero river basin (Costa Rica)

presente, del año 2001) muy precisas acerca de cómo contribuían las coberturas artificiales en la formación de islas de calor urbano en el pasado.

\section{Referencias}

Atkins P. \& Jones, L. (2006). Principios de Química. Los caminos del descubrimiento. Ed. ${ }^{\text {ta }}$ ). Madrid. España: Médica Panamericana. ISBN: 978-950-06-0080-4.

Barsi J., Barker J. L. \& Schott, J. R. (2003). An Atmospheric Correction Parameter Calculator for a Single Thermal Band Earth-Sensing Instrument. International Geoscience and Remote Sensing Symposium (IGARSS). 5. 3014 - 3016 vol.5. 10.1109/IGARSS.2003.1294665.

Barreto, Á., Arbelo, M., Núñez-Casillas, L., Hernández-Leal, P., González-Calvo, A. \& Alonso-Benito, A. (2009). Estimación de la emisividad espectral con datos térmicos ASTER de la Isla de Tenerife. IEEE International Geoscience \& Remote Sensing Symposium, IGARSS 2009, University of Cape Town, South Africa

Becker, F. \& Z.-Li. (1990). Temperature-independent spectral indices in thermal infrared bands, Remote Sens. Environ., 32, 17- 33.

Berk, A., Anderson, G. P., Acharya, P. K., Chetwynd, J. H., Bernstein, L. S., Shettle, E.P., Matthew, M.W. \& Adler-Golden, S. M. (1999). MODTRAN 4 user's manual. Air Force Research Laboratory, Space Vehicles Directorate, Air Force Materiel Command, Hascom AFB, MA.

Carlson, T. \& Ripley, D. (1997). On the Relation between NDVI, Fractional Vegetation Cover, and Leaf Area Index. Remote Sensing of Environment. 62. 241-252. 10.1016/S0034-4257(97)00104-1.

Chander, G., Markham, B. L., \& Helder, D. L. (2009). Summary of current radiometric calibration coefficients for Landsat MSS, TM, ETM+, and EO-1 ALI sensors. Remote sensing of environment 113: 893-903. Coll, C., Galve, J., Sánchez-Tomás, J. \& Caselles, V. (2010). Validation of Landsat-7/ETM+ Thermal-Band Calibration and Atmospheric Correction with Ground-Based Measurements. Geoscience and Remote Sensing, IEEE Transactions on. 48. 547 - 555. 10.1109/ TGRS.2009.2024934.

Denyer, P., Aguilar, T. \& Montero, W. (2014). Cartografía geológica de la península de Nicoya. Universidad de Costa Rica. 
Gillespie, A. R., (1985). Lithologic mapping of silicate rocks using TIMS, in The TIMS Data Users' Workshop, JPL Publ., 86-38, 29-44

Gillespie, A. R., Rokugawa, S., Hook, S. J., Matsunaga, T. \& Kahle, B. (1999). Temperature/emissivity separation algorithm theoretical basis document, version 2.4. Contract NAS5-31372, Natl. Aeronaut. and Space Admin., Washington, D. C.

Niclòs, R., Estrela M. J., Valiente J. A. \& Barberà M. J. (2010). Análisis de correlaciones entre la temperatura del aire y la temperatura de las superficies vegetadas medida con radiometría térmica. Revista de Teledetección, 34: 36-43

Pérez, C. y Muñoz, A.L. (2002). Teledetección: nociones y aplicaciones. Universidad de Salamanca, España.

Prata, J. A. (1994). Land surface temperatures derived from the advanced very high resolution radiometer and the along-0track scanning radiometer: 2. Experimental results and validation of AVHRR algorithms. Journal of Geophysical Research, 99, NO. D6, P. 13,025-13,058.

Quattrochi, D. A., \& Luvall, J. C. (2000). Thermal remote sensing in land surface processes. Boca Raton, FL, USA, CRC Press.

Sobrino, J. A., Sobrino, J., Raissouni, N., Kerr, Olioso, López, M. J., Belaid, El-Kharraz, J., Cuenca, J. \& Dempere (2000). Teledetección. 10.13140/2.1.2883.3283.

Sobrino, J. \& Jiménez-Muñoz, J. C. \& Paolini, L. (2004). Land surface temperature retrieval from LANDSAT TM 5. Remote Sensing of Environment. 90. 434-440. 10.1016/j.rse.2004.02.003.

Valor, E. y Caselles, V. (1996). Mapping Land Surface Emissivity from NDVI: Application to European, African, and South American Areas. Remote Sensing of Environment, 57, 167-184. 